\title{
Updates from the OVRO-LWA: Commissioning a Full-Duty-Cycle Radio-Only Cosmic Ray Detector
}

\author{
Kathryn Plant, ${ }^{a, *}$ Andres Romero-Wolf, ${ }^{b}$ Washington Rodrigues de Carvalho, ${ }^{c}$ \\ Konstantin Belov ${ }^{b}$ and Gregg Hallinan ${ }^{a}$ \\ ${ }^{a}$ California Institute of Technology, \\ 1200 E California Blvd, Pasadena, California, USA \\ ${ }^{b}$ Jet Propulsion Laboratory, \\ 4800 Oak Grove Dr, Pasadena, California, USA \\ ${ }^{c}$ Radboud University, \\ Houtlaan 4, 6525 XZ Nijmegen \\ E-mail: kp@astro.caltech.edu, andrew.romero-wolf@jpl.nasa.gov, \\ carvajr@gmail.com, konstantin.v.belov@jpl.nasa.gov, gh@astro.caltech.edu
}

The Owens Valley Radio Observatory- Long Wavelength Array (OVRO-LWA) in Eastern California is currently undergoing an expansion to 352 dual-polarization antennas and new signal processing infrastructure. The upgraded array will operate a full-duty-cycle cosmic ray detector simultaneously with a variety of radio astronomy observations. Expanding the methods introduced in a previous demonstration, this detector will operate on the radio signals alone to trigger data capture, identify cosmic rays in the presence of radio-frequency interference (RFI), and reconstruct the air shower properties: energy, direction, and $X_{\max }$. When fully commissioned, the OVRO-LWA will observe thousands of cosmic rays per year at energies $10^{17}-10^{18} \mathrm{eV}$ and will constrain the cosmic ray composition across the cosmic ray spectrum's second knee with a typical $X_{\max }$ precision better than $20 \mathrm{~g} / \mathrm{cm}^{2}$ per air shower, thereby offering new composition information across the energy limits of Galactic accelerators. Commissioning for the OVRO-LWA is ongoing and is planned for completion in late 2021. I will present the trigger design, RFI flagging strategy, and a progress update from early commissioning.

$37^{\text {th }}$ International Cosmic Ray Conference (ICRC 2021)

July 12 th - 23rd, 2021

Online - Berlin, Germany

\footnotetext{
${ }^{*}$ Presenter
} 


\section{Introduction}

The flux of cosmic rays incident on Earth likely transitions from Galactic to extragalactic sources at energies between $1 \mathrm{PeV}$ and $1 \mathrm{EeV}$, as evinced by gradual breaks in the cosmic ray spectrum near 1 and $100 \mathrm{PeV}$, increasing anisotropy pointing away from the Galactic plane beyond $8 \mathrm{EeV} \mathrm{[1]} \mathrm{[2],} \mathrm{and} \mathrm{changes} \mathrm{in} \mathrm{composition} \mathrm{above} 1 \mathrm{EeV} \mathrm{[3],} \mathrm{[4].} \mathrm{Current} \mathrm{models} \mathrm{of} \mathrm{shocks} \mathrm{in}$ supernova remnants do not account for the Galactic cosmic ray flux at this transition energy range, requiring an additional class (or classes) of Galactic accelerators at high energies (e.g [5] and references therein).

Resolving additional composition features across the transition is key to disentangling source classes. For cosmic rays observed from the ground as air showers, measuring the column depth of the shower maximum, $X_{\max }$ probes composition. Mean $X_{\max }$ is roughly proportional to the mean of the log of the atomic mass number. Different experimental techniques- involving air fluorescence telescopes, surface particle detectors, and radio antennas- have different systematics, resulting in tensions in the published measurements of $X_{\max }$ vs. energy (e.g. [6], [7]). Additional measurements are needed.

We describe a cosmic ray detection experiment under development as part of an upgrade to the Owens Valley Radio Observatory Long Wavelength Array which will use radio signals alone to trigger data readout, distinguish cosmic ray air-showers from a background of radio frequency interference, and reconstruct shower energy and $X_{\max }$. Reconstructing shower energy and $X_{\max }$ from radio data alone would reduce the dependence of the result on uncertainties in hadronic models. Furthermore, triggering data readout on radio signals without reference to particle scintillators eliminates any systematics due to the scintillators' selection effects as well as making it possible to instrument a large detector area at lower cost. Monroe et al. 2020 [8] demonstrated radio-only cosmic ray observations by detecting 8 cosmic rays during 40 hours of observing with the OVROLWA prior to the upgrade. This experiment demonstrated the radio-only detection technique but required reprogramming the FPGA firmware for the duration of the observation. The cosmic ray detector for the upgraded array will observe with nearly full duty cycle, running alongside other science observations. Section 2 describes the OVRO-LWA, section 3 describes the cosmic ray detector firmware, section 4 discusses radio frequency interference mitigation, and section 5 concludes.

\section{The Owens Valley Radio Observatory Long Wavelength Array}

The Owens Valley Radio Observatory Long Wavelength Array (OVRO-LWA, see Figure 1) is in Eastern California in a deep valley between two mountain ranges, which help shield the array from radio signals from major cities. The OVRO-LWA is currently undergoing an upgrade from 256 dual-polarization dipole antennas to 352 dual-polarization dipoles antennas, which will observe from $12-85 \mathrm{MHz}$ with sensitivity dominated by Galactic noise. The science goals of the OVROLWA include studying space weather events for planets around other stars, stellar and solar flares, and the cosmic dawn epoch.

The cosmic ray detector will share the antennas and electronics used by the other science operations of the array, with different science modules operating simultaneously. The array layout 

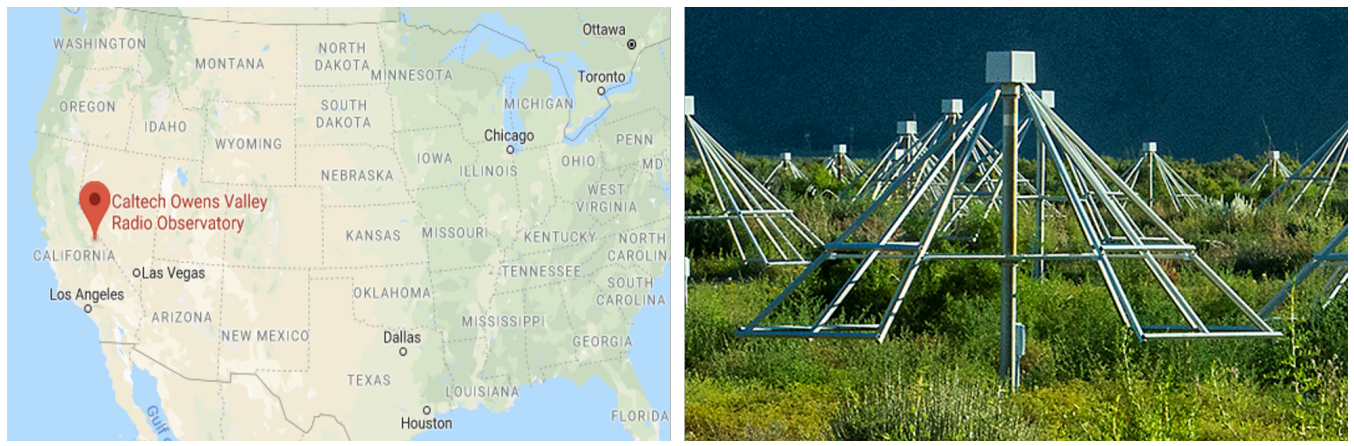

Figure 1: Left: Location of the Owens Valley Radio Observatory (Google map). Right: OVRO-LWA antennas (Photo credit: Gordon Wiltsie).

is optimized for astronomical imaging and consists of a dense core of irregularly-spaced antennas surrounded by sparsely-spaced antennas out to a maximum separation of $2.4 \mathrm{~km}$. Most cosmic rays will be detected within the core of the array, although the sparser regions will be sensitive to inclined showers at higher energies. Figure 2 shows the array layout with a simulated airshower overlaid.

Radio frequency signals from all the antennas will be brought to a central location for digitization, using coaxial cables for shorter distances and optical fiber for longer distances. The signals are sampled with 10-bit ADCs at a sample rate of $196 \mathrm{Msps}$. The first stages of digital signal processing are performed on 11 field programmable gate array (FPGA) boards, which are the second generation of the SNAP2 ${ }^{1}$, containing Xilinx Kintex Ultrascale+ FPGAs and four $40 \mathrm{~Gb}$ ethernet ports. Each SNAP2 processes signals from both polarizations of 32 antennas.

\section{On-FPGA Cosmic Ray Detection Approach}

The cosmic ray detection firmware will operate in parallel on the same FPGAs as the firmware that computes spectra for other astronomy applications (see figure 3), and cosmic ray candidates will be processed in real time in order for observations to run continuously. Since the cosmic ray detector for the upgraded array will run full-time alongside the other observations, and since the upgrade replaces all the signal processing electronics, we build on the technique of [8] but write all new firmware and software. We use FPGA Block RAM (BRAM) to buffer $20 \mu$ s of raw ADC output with $5 \mathrm{~ns}$ time resolution (4096 samples), with the BRAM implemented as a circular buffer which continuously overwrites old samples so that it always contains the most recent $20 \mu$ s of data. Detection of a trigger (see below) halts writing to the buffer, and the $20 \mu$ s timeseries is read out into UDP ethernet packets and transmitted from a QSFP+ ethernet port to a dedicated computer via the same $40 \mathrm{~Gb}$ ethernet switch that also serves the astronomy correlator.

\footnotetext{
${ }^{1}$ https://github.com/casper-astro/casper-hardware/blob/master/FPGA_Hosts/SNAP2/README.md
} 

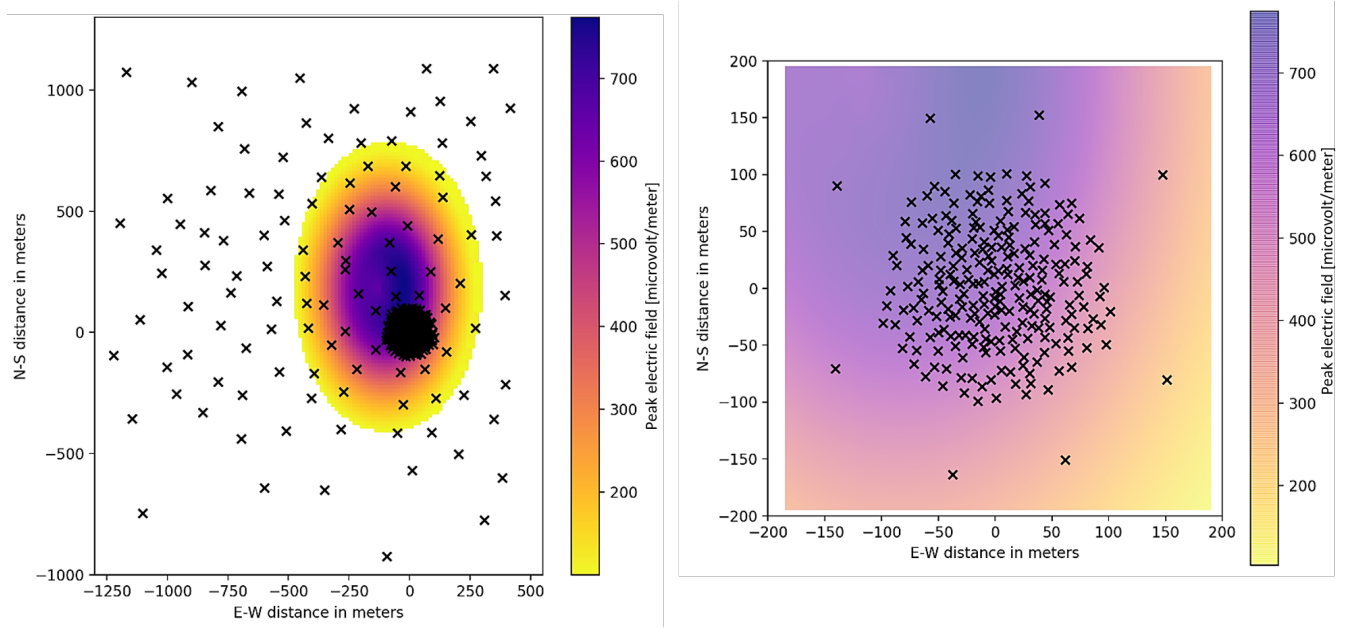

Figure 2: OVRO-LWA array layout overlaid with peak electric field from a simulated 100-PeV proton airshower arriving from the North at 50 degree inclination, simulated with Zhaires for $X_{\max }=695$. The color indicates the peak electric field. The X's mark positions of antennas. Left: all OVRO-LWA antennas. Right: core array.

In parallel with writing to the BRAM buffer, the time series are filtered and thresholded to search for pulses. The pulse detector logic outputs a Boolean 1 when the signal exceeds a threshold. The firmware counts the number of antennas that detect the pulse by extending the Boolean pulses according to the light travel time between the antennas and then summing across antennas. For a cosmic ray to be detected, a threshold number of antennas processed by an individual FPGA must detect the pulse.

All 11 SNAP2 boards will be wired to each other in a loop linking the FPGAs' General Purpose Input Output Pins. Whenever the firmware on one SNAP2 board begins reading out its buffer, it transmits a signal to the other 10 SNAP2 boards to ensure that the processing computer receives a snapshot from the entire array. The initial trigger detection must occur within the subset of antennas that an individual FPGA processes, but any FPGA can trigger the rest of the array to read out data. After a $20 \mu$ s snapshot is read out over ethernet, the FPGA resumes writing to the buffer and awaits new triggers. The firmware is developed and compiled using tools developed by the Collaboration for Astronomy Signal Processing and Electronics Research (CASPER, see e.g. [10]).

\section{Identifying Cosmic Rays Amid Radio Frequency Interference}

Dead time to read out the buffer is limited by the data rate that the receiving computer can handle, not the rate that the boards can transmit. This is because snapshots from all 11 boards must be received by one computer in order to begin classifying the snapshot as either a real cosmic ray, thermal noise, or radio frequency interference (RFI). The receiving computer has two $40 \mathrm{~Gb}$ network interface cards, to receive data from all 11 SNAP2 boards via a switch. To keep the data rate below the limit that the receiving computer can handle, each SNAP2 board must read out its buffer over $920 \mu \mathrm{s}$. 

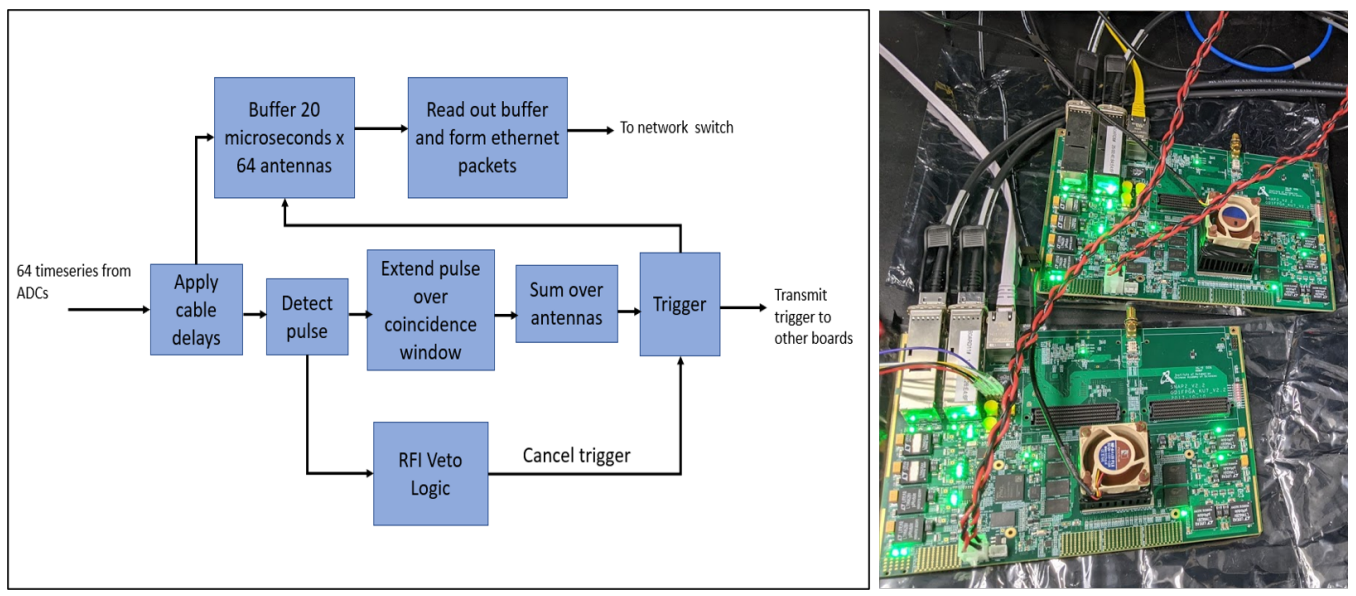

Figure 3: Left: Block diagram overviewing the signal processing for the radio-only trigger. Right: Image of two of the 11 SNAP2 boards that will perform this processing. QFSP+ ports on the left, connected to $40 \mathrm{~Gb}$ ethernet cables transmit buffered timeseries to a central computer when a trigger occurs. Digitizer cards (not pictured) will connect to the four empty FPGA-mezzanine-card connectors on each board. The Xilinx Kintex Ultrascale FPGA is under the fan near the middle of the board.

Monroe et al. 2020 encountered impulsive RFI at a rate of $500 \mathrm{~Hz}$. This background necessitates building a first stage of RFI rejection into the firmware on the FPGAs. The cosmic ray detector for the upgraded array will use all the antennas, not only the two-hundred-meter-diameter dense core that the previous demonstration used, which allows us to use the distant antennas to flag RFI.

Each FPGA processes signals from both polarizations of 32 antennas and the cosmic ray module can determine how antennas are grouped to FPGAs, since this grouping does not affect the other science goals. Antennas in the dense core of the array are divided into nine regions that are each processed by one FPGA, so that neighboring antennas are processed by the same FPGA. These groups are arranged to improve the rate at which a single FPGA will have enough antennas within an air shower footprint to detect the airshower. Additionally, each FPGA will process signals from a few distant antennas up to $2.4 \mathrm{~km}$ away, which act as an RFI veto. These antennas would lie outside the radio footprint of cosmic rays whose shower axis is near the core for the energies we are interested in, and so radio impulses detected by both the core antennas and the very distant outliers can be rejected as RFI. This first stage of RFI flagging has the purpose of removing enough RFI to reduce the trigger rate (thereby reducing the dead time) to a manageable level. More thorough RFI flagging will be performed in later signal processing stages with a CPU. Figure 4 shows the array layout with antennas color-coded by FPGA, with veto antennas marked. Most of the sparse area of the array is grouped to two FPGAs, which will be more sensitive to higher-energy cosmic rays than the core of the array.

Without the distant antennas, [8] used a combination of blocking repetitive events and varying thresholds to reduce the RFI rate to $50 \mathrm{~Hz}$, and their $1.4 \mathrm{~ms}$ readout time corresponded to a $13 \%$ detector dead time. By using distant antennas as vetos, we will maintain constant thresholds, and using the full array expands our detector area. Furthermore, with our faster readout time we expect $4.6 \%$ dead time. 


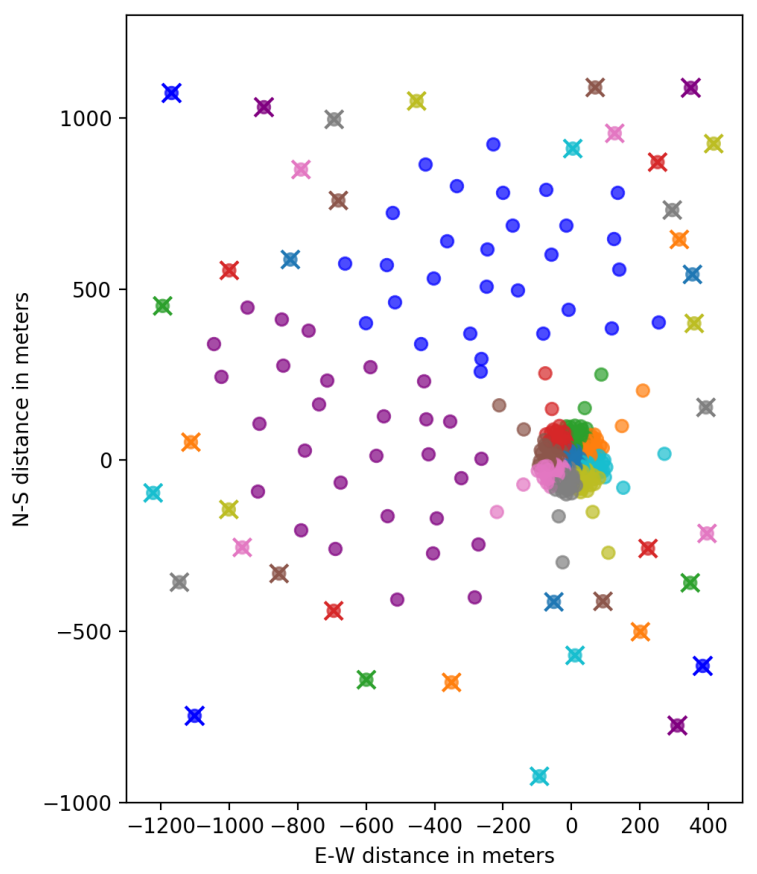

Figure 4: Array layout with antennas color-coded according to their grouping to the FPGAs. Each FPGA processes signals from both polarizations of a group of 32 antennas. Antennas marked with x's are used to veto triggers. Antennas marked with circles are searched for pulses in order to generate triggers.

The main source of impulsive RFI at the Owens Valley Radio Observatory has been faulty power lines from nearby small towns. Over the last few years, the observatory undertook an effort to identify the specific locations of the problems, which the power company then repaired. This will greatly improve the RFI environment for the upgraded OVRO-LWA, although on-chip RFI flagging will remain necessary.

After candidate cosmic ray events are detected by an FPGA, triggering all FPGAs to read out their $20 \mu$ s buffer, candidate cosmic rays will be classified with several stages, with code running on a CPU, based on the approach taken by [8]. First, signals from each antenna will be filtered, identifying the power and arrival time of the pulse. The pulse arrival times will be fit to a simple model of an arriving wavefront. Events that fit poorly will be flagged as thermal coincidences and discarded. The wavefront fit gives the arrival direction, and events from a few specific directions of known RFI sources will be discarded. The main source of RFI that can pass these criteria will likely be from airplanes. Candidate events will be checked for clustering in time along airplane tracks. We expect a few tens of events per day to remain, and these will be compared to simulated airshower footprints (both in lateral power distribution and polarization signature) to identify genuine cosmic rays, as successfully demonstrated in Monroe et al. 2020. We know of no source of RFI that would 
produce emission with the beaming and polarization properties of a cosmic ray.

\section{Conclusions and Future Outlook}

The proceedings describes an ongoing upgrade to the OVRO-LWA, expanding on a previouslydemonstrated technique that uses radio signals alone to trigger data readout, reject radio frequency interference, and reconstruct shower properties. When all antennas are available, we expect to detect thousands cosmic rays per year in the energy range $100 \mathrm{PeV}-1 \mathrm{EeV}$. We plan to estimate $X_{\max }$ for each cosmic ray by comparing the recorded signals to radio emission from air-showers simulated with ZHAIRES. Based on simulations we expect to estimate $X_{\max }$ with precision better than $20 \mathrm{~g} / \mathrm{cm}^{2}$ for ideal events. We are also exploring using the interferometric back-propagation technique developed by Schoorlemmer and Carvalho 2020 [9]. The sample of cosmic rays with $X_{\max }$ estimates will be used to constrain the composition of cosmic rays from $100 \mathrm{PeV}-1 \mathrm{EeV}$.

Currently, the antenna beam map is known to within $10 \%$. A planned effort aims to map the beams to within one percent, using a low-frequency feed installed on the Owens Valley Radio Observatory's $40 \mathrm{~m}$ telescope. Additionally, we intend to use a small number of scintillators in the future as an extra validation of the radio technique. These would be used for a limited time in part of the core of the array.

After completing the upgrade, we will begin observations in late 2021. The OVRO-LWA will provide a large sample of thousands of cosmic rays per year with $X_{\max }$ measurements, probing composition features across the Galactic to extragalactic transition.

Acknowledgements This material is based in part upon work supported by the National Science Foundation under the NSF Graduate Research Fellowships Program (K. Plant) as well as Grant AST- 1654815 and AST-1828784. We also acknowledge the support of the Caltech/JPL President's and Director's Research and Development Fund.

\section{References}

[1] A. Aab, P. Abreu, M. Aglietta, I. Samarai, and et al. Observation of a large-scale anisotropy in the arrival directions of cosmic rays above $810^{1} 8 \mathrm{eV}$. Science, 357(6357):12661270, 2017.

[2] E. (Pierre Auger Collaboration) Roulet. Large-scale anisotropies above $0.03 \mathrm{EeV}$ measured by the Pierre Auger Observatory. Proceedings of Science ICRC, 2019.

[3] A. Aab, P. Abreu, M. Aglietta, E. J. Ahn, Al Samarai, and et al. Evidence for a mixed mass composition at the 'ankle' in the cosmic-ray spectrum. Physics Letters, Section B: Nuclear, Elementary Particle and High-Energy Physics, 762:288295, 2016

[4] Ru Abbasi, M Abe, T Abu-Zayyad, M Allen, and et al. Mass composition of ultra-high-energy cosmic rays with the Telescope Array Surface Detector Data. Technical report, 2019.

[5] Julia Becker Tjus and Lukas Merten. Closing in on the origin of Galactic cosmic rays using multimessenger information. Physics Reports, 2020. 
[6] Corstanje, A. et al. Depth of shower maximum and mass composition of cosmic rays from 50 $\mathrm{PeV}$ to $2 \mathrm{EeV}$ measured with the LOFAR radio telescope. Phys.Rev.D.103(10). 2021.

[7] F. Schroeder. News from Cosmic Ray Air Showers (ICRC 2019 - Cosmic Ray Indirect Rapport). Proceedings of Science ICRC, 2019.

[8] Ryan Monroe, et al. Self-triggered radio detection and identification of cosmic air showers with the OVRO-LWA. Nuclear Instruments and Methods in Physics Re- search, Section A: Accelerators, Spectrometers, Detectors and Associated Equip- ment, 953(November 2019): 163086, 2020 .

[9] H. Schoorlemmer and W. Carvalho. Radio interferometry applied to the observation of cosmicray induced extensive airshowers. arXiv:2006.10348, 2020.

[10] J. Hickish et al. A Decade of Developing Radio-Astronomy Instrumentation using CASPER Open-Source Technology. Journal of Astronomical Instrumentation, Volume 5, Issue 4, 2016. 